\title{
Coronavirus as a Global Complex Problem Looking for Resilient Solutions
}

\author{
Mohamed Buheji \\ Founder of the International Inspiration Economy Project, Bahrain \\ E-mail: buhejim@gmail.com
}

Received: March 10, 2020 Accepted: March 21, 2020 Published: March 23, 2020

doi:10.5296/bms.v11i1.16730ＵRL: https://doi.org/10.5296/bms.v11i1.16730

\begin{abstract}
A new type of mysterious zoonotic virus called coronavirus is swiping the world, killing thousands of people and infecting hundreds of thousands, without any foreseen innovative solutions. The pandemic of COVID-19 could be categorised to be a complex problem since it is both challenging to discover and difficult to manage. The complexity of the coronavirus comes from its capacity to interact and evolve in unexpected ways. Yet, with this complex global problem, it is still possible to come with innovative solutions, if we managed to follow practices that shorten the time to learn about the virus and comprehend it.

The researcher reviews the sequence of events of the first three months since the breakout of COVID-19 in December 2019, to set the mindset of the reader to consider this a complex problem that has constructs that need to be addressed, before we look for classical solutions. In order to simplify the complexity of this virus as a problem, a framework is proposed to help the specialised scientists to study the puzzle of coronavirus as a time-bound problem that could have resilient solutions. The framework focus on the traceability of the virus, besides its 'doubling time' and the Case Fatality Rate (CFR). Formulas of change and overall effectiveness of problem-solving are discussed, followed by a recommendation of how to optimise the utilisation of the proposed framework.
\end{abstract}

Keywords: Coronavirus COVID-19, Zoonotic Viruses, Complex Problems, Problems Solving, Case Methods, Traceability, Resilient Solutions.

\section{Introduction}

COVID-19 and its impact is now a complex global problem looking for a resilient solution that addresses the problem beyond its current context, i.e. a public health crisis. The resilient solution could come from defining, codifying, classifying and then stratifying the variables or 
the constructs of this life-threating complex problem. Then, based on understanding these variables, we can determine the resources and the strategies needed. Buheji (2018), McGrath and Sargut (2011).

The events of the coronavirus, since its outbreak for the first three months, are reviewed and synthesised. COVID-19 outbreak is explored as a zoonotic problem, then the vaccine development is clarified as a limited corrective not preventive solution for this problem. The novel challenge of the coronavirus problem and how it is influencing the psychology of the people is discussed. The complexity of the COVID-19 is mentioned in relevance to the need for the case method as an approach for such a complex problem. Wu et al. (2020), Drohan (2020).

The author shows that the common solutions for such a complex global problem are quarantine, social isolation, treatment of difficult cases, and searching for medicines or vaccine to treat or prevent people from the virus. However, knowing the nature and the history of the zoonotic virus and their ability to modify itself, such a solution are not sustainable.

The paper identifies specific linkages that go with the spread of this virus to be embedded in the mindset of the researchers. These linkages are reflected in a proposed framework that focuses on the traceability of the virus and its related symptoms. Also, we study the virus 'doubling time' and the Case Fatality Rate (CFR). The target of this work is to prepare the world for the coming future foresighted challenges by bringing scientists together to see the COVID-19 complexity and solutions from different perspectives that would bring more holistic breakthroughs.

\section{Literature Review}

\subsection{Reviewing the Events of the Coronavirus (in first three months)}

The complexity of zoonotic viruses' outbreaks is increasing in both their capacity to cause severe infections, epidemics that goes to be global and become pandemics, besides creating disruptions to human quality of life and socio-economic status. These Zoonoses diseases and infections naturally transmitted between people and animals. These zoonotic nature outbreaks come in three forms: endemic zoonoses that make the virus present in many places and affect many people and animals, epidemic zoonoses which are sporadic in temporal and spatial distribution; and emerging and re-emerging zoonoses which are newly appearing in a population or have existed previously but are rapidly increasing in incidence or geographical range, as per WHO (2019). So far, almost all the solutions offered in literature been coming from one main discipline, medicine.

Before the outbreak of the COVID-19, the world had different chances to bring resilient solutions to such outbreaks when it faced Severe Acute Respiratory Syndrome (SARS), Swine Flu H1N1 2009, Yellow fever, Avian Influenza (H5N1) and (H7N9), West Nile virus and the Middle East respiratory syndrome coronavirus (MERS-CoV), but it didn't. There is great similarity between all of these viruses that could bring a hint to their both complexity and resilient solutions. This latest version of coronavirus, for example, has a great similarity with 
(SARS) which occurred in 2002-2003. However, the SARS is thought to have originated in bats, the virus jumped to humans, becoming a global epidemic across 37 countries before contained through infection control practices.

In analogy to SARS, the COVID-19 series of incidence started on December 31st, 2019. The Chinese health officials detected a new strain of the coronavirus in a Wuhan food market. The Huanan Seafood Market sold wild and exotic animals less than a mile from the Hankou train station through which at 100,000 passengers flowed each day. On January 1st, Chinese authorities closed the market. On January 9th, Chinese researchers identified a new strain of the coronavirus. In the same month, $41 \%$ of the identified patients in Wuhan, infected other patients or other care providers.

On January 30th, World Health Organization Director Dr Tedros Adhanom Ghebreyesus declared a public health emergency. By February, Beijing authorities claimed to have locked down 50 million Chinese in order to contain outbreaks. This started to affect the supplies to and from China. It is estimated more than one-third of China's manufacturing and services are affected. This began to escalate to many countries and organisations where their sales, income and growth dependent on Chinese supplies. WHO (2020b)

Several news accounts report companies and medical researchers rushing into action. To meet the demand, a number of firms are increasing the production of protective masks and suits. Researchers collaborated across global networks to develop an anti-virus.

\subsection{COVID-19 as a Zoonotic Problem}

The virus is known as COVID-19 — short for Coronavirus disease 2019 - and it has spread around the world, infecting more than 89,000 people and killing more than 3,000 till the moment of writing this paper. The "Coronavirus" comes from a class of viruses, which the latest outbreak was seen in SARS. It is also from similar family as the Middle East Respiratory Syndrome (MERS) of 2012 and 2014-2017, which may have started in camels and spread to humans. These viruses have a crown-like shape with a spike of proteins on top. They carry mostly the same symptoms including fever, coughing and shortness of breath, on a level much more serious than that seen in cold sufferers. But, in COVID-19, these symptoms are severe. Wu et al. (2020).

Coronaviruses spread when the spike proteins on their surface bind tightly to receptor proteins found on the surface of human cells. This is causing the virus to have strong spread and to be more complex. Its complexity comes from being actively infectious to the human cells and could replicate very fast. McGrath and Sargut (2011).

Thus, the COVID-19 is a type of viruses that need hosts to live and propagate. Their complexity increases over time as they develop variable characteristics. Genome analyses of this virus indicate that the novelty of it is that it has a unique capability to manifest itself in animals. This coincides with the latest study published in The Lancet, which emphasised that Zoonotic infectious diseases are on the rise. Such virus has a reproductive factor of more than 
2.68, which means that the virus has the capacity to double its reach in every 6.4 days. Roser and Ritchie (2020).

This virus even capable of having more than this reproductive factor due to the epicentre of the outbreaks is being China, and the frequency of travel all over the world is now at its most. Just at the time of writing the paper and after less than three months from the outbreak, the virus is now available on more than 70 countries with different extent. In total, $3 \%$ of those infected with this virus died. This means this virus is a deadly one and especially if it hits the older population. Wu et al (2020).

\subsection{Development of a Vaccine as a Solution}

The experience from the 2003 SARS outbreak is that it took the world about 20 months to get a vaccine ready for human trials. Then the vaccine was never used, because the disease was contained by that time. The coronavirus vaccine is still also more than four months to a year away, despite the development of genomics technology. The collaborations of research labs around the world, yet did not help to have the anti-COVID-19 vaccine be available today for all those humans that need it. This anti-virus vaccine still needs special processing, and going to be both expensive and risky. i.e. Many of these vaccines has been recalled due to not being fit to fight to this self-evolving virus. Hemel and Ouellette (2020).

While the researchers are still striving to conduct extensive testing to prove the COVID-19 vaccine is safe and effective, we should work as thinkers and community change leaders to explore other alternative solutions for the problem. Therefore, one could sum here that development of the vaccine is not the ultimate solution to contain the problem of COVID-19 or any other similar problems, we need more alternative solutions that make people and businesses come back to life more actively.

\subsection{The Novel Challenge of the Coronavirus Problem}

In December 2019, four people showed up in a hospital in Wuhan, Hubei Province, China, population 11 million. Each patient had a fever, shortness of breath and other pneumonia-like symptoms. By early February 2020, the virus had infected over 40,000 people of whom more than 1000 died. Khan (2020), Roser and Ritchie (2020).

The novel coronavirus is that it is an open-ended problem intended to force decision-making that produces alternative courses of action. The effectiveness of this problem-solving method comes from its particular questions. These questions help us to analyse or evaluate assumptions, logic, and evidence. This called 'question typology'. Zhu et al. (2020).

The main challenge is that the vaccine of anti-coronavirus is not available and taking a long time to come compared to the spread of the corona. Hence, this novel ongoing epidemic requires many preparations to deal with. It requires open-ended approaches to solving such a complex problem. Hemel and Ouellette (2020).

Bill Gates (2020) emphasis that COVID-19 is a type of crisis that need two equally important responsibilities: a problem to be solved immediately and then a problem that should be 
eliminated from occurring again to avoid more complex and long-term consequences. In order to improve our ability to respond to outbreaks, similar to COVID-19, which are surely going to increase in damage and frequency we need to prepare in a different way, especially we are living in an environment that would help to transmit it efficiently. The average infected person spreads the disease to two or three others - an exponential rate of increase, Gate (2020).

\subsection{The Psychology of Coronavirus Problem}

History of humans tell us that we are great at solving urgent, immediate problems, but we are so slow in dealing with the foresighted ones. COVID-19 is one such problem that brings it with new waves of infectious viruses' threats. Eliminating coronavirus and similar humans' threats go beyond immediate solutions. It beyond stocking disinfectants and surgical masks along with hand-gloves, or increasing the isolation beds or areas, or training the public continuously for handwash. Even the issue of COVID-19 is beyond having a vaccine. Gates (2020).

The psychology of human beings, in general, equip them to be the best problem-solvers for short term solutions. However, most of us have a mindset that is not equipped for a challenge that requires long term solutions, especially if the solutions do not come from the specialities concerned about the problem, but extracted from the interpolation of the multi-disciplinary perspectives. Wetti (2020).

A complex old evolving problem as the coronaviruses, cannot come from the speciality of medicine only. This what history tells us for any complex solution that humanity tackled before. Therefore, we need to bring solutions that come from within us.

Different countries have taken different levels of countermeasures since the speed of the escalation of the virus and the way it is escalating rang the highest alert bill of the WHO emergency level. Many countries have taken measures that restricted travelling only to essential business, providing health-related items, discontinuing ongoing trips, limiting travel outside of home and office. This increased the anxiety of the majority of the world population and especially where the virus reached. WHO (2020a).

\subsection{Complexity of COVID-19 Problem}

Complex problems always come with featured that are unpredictable, evolving, surprising, and with high throughput emergent behaviour. Besides, it can come from unexpected corners or totally hidden side or perspective. COVID-19 shown to have many interconnected features and symptoms, yet it also carries interdependent characteristics and this what makes it more complex than any other previous breakout.

COVID-19 being a more complex problem than complicated, as it is a problem with many components, and that what makes it more challenging to manage. With this virus power coming from its intrinsic capacity, it is harder to predict what it could do next since it is like a complex system that interacts in unexpected ways. The emergent properties and the behaviour of this new coronavirus with its unique capacity for self-organisation makes its 
non-deterministic, regardless of the amount of the speciality efforts we put into it. Thus, with this virus, it is not hard to make sense of things; this means it needs practices that shorten the time to learn and comprehend things. Roser and Ritchie (2020).

The COVID-19 complexity should make us, as problem trouble-shooters, to consider that its past behaviours may not necessarily predict its future behaviour. With a complex problem like this, its surprises are often more significant than its average past. Collectively we can do a good deal to navigate and mitigate the risks of this complexity; however, this thinking has not permeated yet in the mind of the researchers and the scientists who are researching for specialised solutions, as anti-viral vaccines. Buheji (2018).

The advice thus is not to manage COVID-19 as a complicated specialised problem, but rather a complex problem to avoid serious, expensive mistakes. The issue and the reason why this virus is not complicated is that it does not have many moving parts, yet it transformed with no pattern. Hence, we cannot depend on predictions, since its interactions with the victim come from different sources. Khan (2020).

The complexity of COVID-19 is confirmed by three characteristics that this virus clearly has made since it came in December 2019:

First, its multiplicity. This virus has a high number of potentially interacting elements. Second, interdependence. This version of coronavirus has unique connected elements that are independent of each other.

Third, its diversity. This virus threat has a degree of heterogeneity.

Thus, the more we have multiplicity, interdependence, and diversity, the more we will see that the virus become complex. This means it is a virus beyond being complicated and thus we cannot benefit from knowing the starting conditions, as most efforts are made now from many scientists, in an intention to use that to predict the outcome.

The complexity of the virus might make each starting condition in every country to produce different outcomes, depending on what it interacted with and what elements enhanced its strength. Therefore, we need also to have the capacity to continually change our reaction and continuously adjust to this virus evolution.

Dealing with such sophisticated human threat, means we need to know the type of decisions that would help us to minimise surprises and expand our understanding of every rare event. Hence here, we need to have resilient systems that have the adaptability of the observed patterns that emerged over time. This resilience should reflect on how we deal with the complexity of the events, which goes to appreciate the rare event that caused the variations.

\subsection{Case Method as an Approach for Complex Problem-Solving}

Drohan (2020) emphasised that the novel coronavirus threat led to an uncertain information environment, which increased the complexity of the problem. This made Drohan suggest the use of 'case method' analysis for solving such global life-threating dilemma. Such method is 
used today in lots of troubleshooting for complex cases due to its investigative thinking style. Christensen et al. (1987).

The approach of the cases method is useful in uncertain situations, similar to the COVID-19 global crisis, or turbulent dynamic environments. The case method triggers a type of reflective open-ended learning that can lead to crucial questions which could produce competitive solutions. The scenarios of cases try to engage participants in learning through facilitating discussions and more interactions between the stakeholders. This dynamic method brings crowd-sourcing solutions in real-time. The approach is an appropriate fit for dealing with challenges and opportunities in complex environments.

Case method helps the participant to consider the severe threats as the coronavirus and the challenges it brings and strives to convert them into opportunities. This method usually encompasses all the information needed about the investigated environment. For example, where the virus occurs more in dry or wet surfaces, in the land, or the sea. What type of air or medium it is transferred through and or where its throughput is most? The case method can identify the most suitable answers to these queries that would lead to eliminate the virus or mitigate its risks.

The case information is usually categorised into four types: functional information, capability information, operational information, and power information. This categorisation then uses a reductionist approach, and hence information becomes units either 0 , or 1 . Thus the information could be seen as one system of interconnected environment and help us to think holistically. This would help us to classify the causalities of the originating observations according to their source: humans, animals, environment, machines, materials, methods and policies. Once the vulnerable are detected at the right time, the causes of risks could be flipped to be sources of opportunities that bring solutions. Christensen et al. (1987).

Since the case method requires us to identify the whole story that helps us to trigger the thoughts of the beneficiaries and integrate them with the problem, it helps us to think about the questions rather than about the answers this facilitates substantive thinking. This maximises the possibility of getting the thread of the problems which would most probably come from the field, and then help develop collaborative solutions. Drohan (2020).

In the case of the COVID-19 crisis, we need to define first the objective of engaging the public or the stakeholders in solving its related issues. Then, we need to identify the linkages, the patterns, the trends, the anomalies, and anticipate emergent conditions that come with the spread of the virus in certain areas, or countries, or environment, or among specific species, type of human category, etc. Then, these linkages need to be analysed further to determine ways to influence the desired change via the information of the capabilities identified earlier. Then activities that influence the capabilities need to be designed to enhance the desired outcomes.

\subsection{Designing Resilient Solutions}


In complex solutions as the COVID-19 crisis, we need a resilient solution that emphasis 'no one size fits all' approach. Hence, each particular measurement variable proposed in a framework of any study should be approached based on the conditions of the targeted beneficiary, or on the context which they are applied in. For example, if a framework is adopted in east Asia, the same framework can be adopted in Europe but with some modification suitable to the context and lifestyle of the region.

Therefore, resilient solutions could come from encountering the current COVID-19 challenges, with a mindset of 'learning by doing' or 'learning by exploring'; however, through following a specific framework. The main resilient solution criteria that the proposed framework should bring is the enhancement of communities and global resistance and functionality. This start with exploring the elements that contribute to interdisciplinary solution designs. The other criteria for resilient solution should tailor to make an accurate and reproducible response and manage the mindset of the stakeholders of seeing the alternatives and the hidden opportunities inside the COVID-19 challenge, such as preparing the world communities towards a better future. Buheji (2020).

\section{Methodology}

In order to support the selective analysis of the coronavirus outbreak through complex case methods, as mentioned in the literature review, this paper targets to identify specific linkages among the major variables that go with the spread of this virus. Such linkages should support the efforts and the mindset of the researchers to eliminate or contain such complex viruses.

The methodology followed here is built to help answer future questions that focus on the opportunities hidden within such complex viruses and which can be divided into two questions:

1 What are the patterns we need to enhance or eliminate that would strain the linkages of the virus?

\section{What Are the Early Trends and Anomalies That Would Be of Pertinent with Virus Spread?}

In order to simplify the complexity of this virus as a problem, a framework is proposed to help the specialised scientists to study the puzzle of coronavirus as a time-bound problem. The framework focus on the traceability of the virus and its related symptoms. Also, we study the virus 'doubling time' and the Case Fatality Rate (CFR). Eddy (2020).

The outcome of this methodology targets to help the scientists to define the variables that they need to be investigated, or eliminated in this virus problem. The idea is either to be more ready for the coming viral threats, or see the hidden opportunities on those relations or linkages that can enhance our competency to eliminate or mitigate such complex viral risks once they outbreak again.

\section{Proposed Generic Framework for COVID-19 Problem}


4.1 Management of Change in the COVID-19 Complex Problem

As discussed in the literature review, and taking into consideration the warning of Bill Gates (2020) we need to bring resilient solutions that change our approach to the problem and in the same time prevent its occurrence, even in the different form again. Hence, dealing with a complex problem as COVID-19 requires preventative measures that eliminate any causality for such incidences and sustenance of such elimination for such problem not occur in the future. This means there should be a change management program that uses the formula of change to help us reach the desired change of permanent elimination of such a complex problem.

\section{Achieving the Desired Change $=D x M x P$}

Where D stands for the level of dissatisfaction of the situations that led or might be leading to another Coronavirus or similar outbreaks. M stands for the model that can be created that can be waste-free, i.e. no virus linkages or relations left without being either modified, eliminated, or utilised for the benefit of a safer environment.

This change formula can help the scientists to study any proposed solutions, taking into consideration the framework proposed in Figure (1) where traceability, time-bound and CFR would part of the $D$ (the measure) and part of $M$, i.e. the (targeted solution).

Once $\mathrm{D}$ and $\mathrm{M}$ are created as part of the framework of the solution, then, $\mathrm{P}$, which stands for the process of managing change towards the optimal desired condition can be triggered. Here, we need to use the optimisation of constraints, i.e. see the areas where we can eliminate the causes of the instability of dealing with the dangers of the virus linkages, or its relations and try to modify them by taking effective action plans. Taking such actions should reduce the strain on the healthcare staff and make them safe and proactive, besides make the scientists being selective in their studies, screening, diagnosis and treatment approaches or protocols.

To optimise the $(\mathrm{P})$ process of change, to deal with the fast-spreading virus, we need to use a formula that measures the effectiveness of the process of change.

\section{Effectiveness of Process Change = Availability $x$ Quality x Efficiency}

Through this formula, we can optimise our availability for constraining the linkages or the relations that might strengthen the possibilities for COVID-19 presence, i.e. controlling the virus time-bound. We can also enhance the quality of the traceability of the virus and thus strengthening our capacity to take preventive measures to avoid the breakout spread or coming back again. Then, the efficiency of managing the virus is measured by the doubling time.

The above two formulas should help in achieving more effectiveness changing the outbreak threat and eliminating its continuous threat to humanity.

\subsection{Coronavirus as a Time-bound (Availability) Puzzle}

When humans infected with the disease of COVID-19, all the healthcare workers start traceability and tracking and competition with time. The efforts to retrace all the step paths of those infected patients are so vital to the patient, the patient relatives, the community, the 
country; besides it is vital for the continuation of the existence of humanity today. The uniqueness of the threat of the COVID-19 as a puzzling problem is even beyond the Spanish Flu in 1918, as it has the combination of both being infectiousness and causes severe destruction of its victims, besides it a problem that has other motivators in today open skies and advanced consumers market. The possibility of it being transferred by one passenger from one country, or even one continent to another in less than a few hours is higher than ever. Eddy (2020).

Any tracking achievement, higher availability, could prevent countless lives and would stop this deadly virus from further spread. However, many of the coronavirus patients become so ill and would be placed on a respiratory ventilator with no capability to cooperate with the authority's investigations. Therefore, we need to develop urgently more effective traceability methods that could retrace the patients and then be ahead of their infectious possibilities, in order to contain it and eliminate it permanently.

If we want to stop the spread of the COVID-19, we need first to enhance our capacity of being available for tracing the victim steps within the fourteen days incubation period. The capacity of this complex investigators needs to be sharpened to be capable of collecting the different pieces of evidence in a short time. These pieces should be shared nationally and internationally through effective communication models.

\subsection{The Traceability (Quality) Challenge}

Today and with the peak of this virus outbreak, many cases are being investigated around the world, but many without successful leads. This traceability becomes a global challenge issue and undermines the quality of the great efforts taken globally, if it not synthesised and seen from different perspectives. Many retracing steps of where the COVID-19 need to be chained so that the investigators would not only know from where the patient has come, but also would know from who, or what, or when he/she got this virus. This shortage of knowledge presents a future opportunity for future researchers.

The repeated incidents show that the more the number of people attending a gathering, the higher the probability that one of them would be infected. However, this would make it even harder to trace how he/she got infected. Currently, we as humans proven to be weak in tracing the incidence of infection in the right time and place, and thus we ended emphasising the only solution to be self-isolation.

Traceability would continue to be a challenge and even more difficult in countries of the boundary of personal freedom is high, as in Europe. Therefore, a need for a system that ensures collective traceability of repeated places and times of incidents is clearly undebatable. The main challenge for tracing this novel virus is that it could be transferred to the victims again from either animals or people. Besides, it has the capacity to spread easily from person to person, but especially in confined spaces. The traceability of the pathogen which can travel through the air, and which can come from virus carriers with no symptoms and which have the virus in when he/she breathes, talks, coughs or sneezes, are challenges that increase the 
problem complex. Therefore, more efforts needed to investigate how we can enhance this traceability. Zhu et. al (2020).

\subsection{The Virus Doubling Time - (the Efficiency Measure)}

In an outbreak of infectious disease, it is important to know not only the number of cases, but also the rate at which the number of cases is changing. A helpful metric to measure the rate of change is the doubling time. This is the time it took for the number of cases to double. If the number of cases is 100 today and it was 50 three days ago, then the doubling time is three days.

The doubling time in a disease outbreak is not constant, and for the outbreak of COVID-19, it has changed in recent weeks and will continue to change with time. What we want to know is the total number of COVID-19 cases. But since not everyone is tested, it is only the number of known cases (confirmed cases + suspected cases) that we can know. Since the coronavirus disease has only mild symptoms for most (see below), it is likely the case that the number of confirmed and suspected cases are smaller than the number of total cases.

Based on the global WHO data up to and including 7th March 2020, the doubling time for COVID-19 is as follows:

Doubling time for the global number of cases (including China): 21 days

Doubling time for the global number of cases (excluding China): 4 days

The exponential growth of the doubling time of the virus means the lower efficiency of investigators to grasp the virus and eliminate its threat at the right time, or with minimal resources. If during the outbreak, the number of cases is doubling, and this doubling time stays constant, then the outbreak is spreading exponentially.

\subsection{Case Fatality Rate - (the Overall Effectiveness Measure)}

There are many diseases that have much higher Case Fatality Rate (CFR) than the COVID-19, For example, the Diabetes CFR amongst the people aged 80 years or older, at $14.8 \%$. CFRs have been higher in people with other medical conditions than in healthy people. In men, the CFR for hypertension patients is $25 \%$ and then is $22 \%$ for women.

More than $10 \%$ of those diagnosed with COVID-19 who already had cardiovascular disease, died as a result of the virus. Diabetes, chronic respiratory diseases, hypertension, and cancer were all risk factors. Most of the data shows that the majority of the dying patients had a CFR of only $0.9 \%$ without a pre-existing health condition. Zhu et al. (2020).

Hence, the current COVID-19 global CFR is still considered to be less than one-fourth of any chronic disease. Similar to other illnesses, it is natural that the CFR for the COVID-19 does not have a single figure since it varies by location, and is typically changing over time. The exaggeration about the severity of the disease is creating unnecessary complication about the problem.

In a period of three months since this virus outbreak started, i.e. till 7th of March 2020, the 


\section{Macrothink}

global CFR for COVID-19 is less than 4\% [based on 101,927 confirmed cases and 3480 deaths]. However, we need to keep monitoring the CFR to measure the overall effectiveness on the human efforts to eliminate or mitigate this complex problem.

\subsection{Generic Framework Proposed}

Clearly, the source of the problem is not discovered or eliminated early; thus, we have low availability of an effective public health inspection on the right time and place. Most recent outbreaks, as SARS and recently COVID-19 are observed in the hospital rather than in the source, i.e. in markets like Wuhan's. Thus, we find that the world has to manage a complicated zoonotic reported outbreak associated with healthcare infections rather than foodborne infections.

This framework proposes that the traceability, time-bound, and CFR are taken into consideration when investigating any complex problem similar to the COVID-19 threat. Figure 1 represents the linkages that can be taken into the mindset of the scientists to help see the problem from different perspectives and hopefully stratify it.

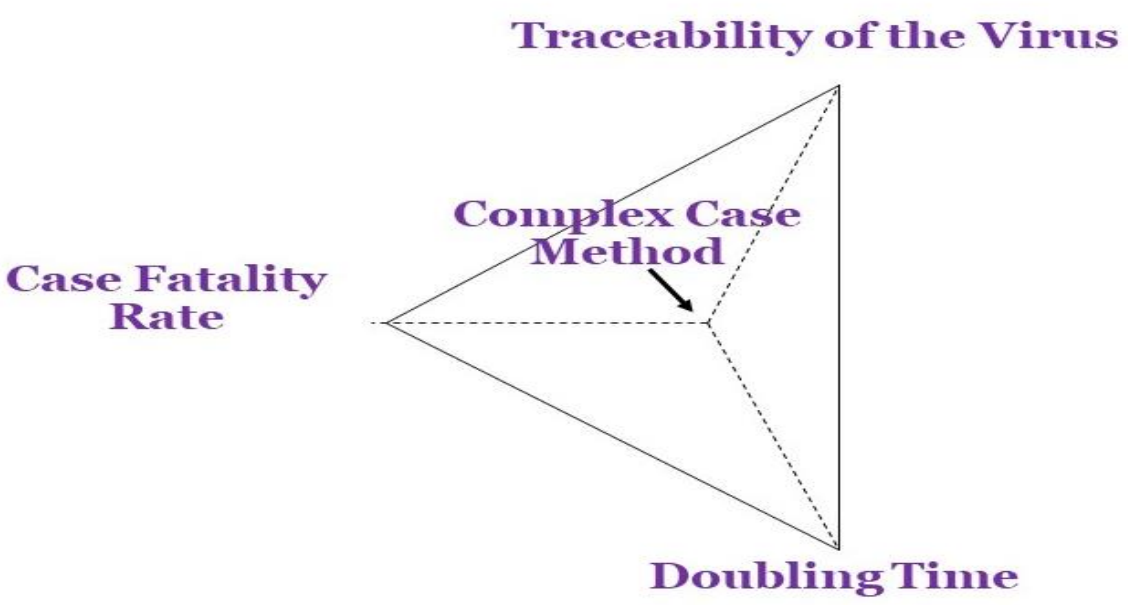

Figure 1. Proposed Framework for Simplifying/Stratifying COVID-19 Problem

The model emphasis that is having the three elements the traceability capacity, the doubling time and the CFR; besides the application of the complex case methods is considered by the scientists and the current leading investigators of the COVID-19, could bring a new better creative solution to humanity and break any mindset barriers.

\section{Discussion and Conclusion}

5.1 COVID-19 as a Complex Problem 
COVID-19 is a complex problem that needs a thoughtful, holistic mindset which manages the constructive chaos that this virus has created. This paper comes to support all the great efforts which have been taken or being taken or would be taken by many great healthcare practitioners and scientists all over the world. The paper opens a new perspective for the researchers to see the complexity of the COVID-19 and similar outbreaks as a complex problem that needs to be stratified from different angles. Therefore, the ultimate goal of this work is to facilitate all the attempts that would address the main challenges of COVID-19 as a dilemma that could bring an innovative idea and a permanent solution for such life-threatening problem and develop the researchers capacity in dealing with such challenges in the future.

The author calls for the need for different characterisation and observations of the COVID-19 pandemic problem environment. The relationships and linkages proposed in the framework can help eliminate and mitigate the virus problem from being present or spreading. This paper warns the world decision makers, scientists and even public health leaders that failing to approach problems of global influence without a resilient multidisciplinary approach framework would continue to cost the world lives, billions of dollars with great economic, psychologic and social pain followed by great disruptions, loss and chaos.

\subsection{Implications and Limitations of the Paper}

This paper carries one main implication which is helping to develop suitable strategies for setting collaborative programs that would proactively tackle future complex problems similar to the new coronavirus through problem-solving models that help to see solutions from a more holistic approach, rather than working on corrective reactive scientific approaches represented by either isolations or vaccination.

The limitation of this paper is that it is not tested on the field yet. Therefore, more research is recommended in this area to debate, support, develop and report the effectiveness of this methodology and mindset.

The author could not also compare this problem-solving proposed approach to other zoonotic diseases to strengthen the evidence of this paper, due it has not even been followed for the earlier diseases. Further studies are recommended to check similar frameworks that could increase the global resistant and functionality in its fighting against current COVID-19 crisis or similar coming threats. Such frameworks need to be considered and modified, taking into consideration the different types of dynamical changes in the field. But the more we test these proposed frameworks, the more we can bring to the world to such resilient unified solution.

\subsection{Final Words}

The world needs to be very humble and accepts many humilities if we are to eliminate such complex zoonotic infectious global diseases, as solutions might not come necessarily from top universities professors, large research labs, or even countries who are leading the fight against this deadly virus currently. We should not see this pandemic from one specialised perspective, but rather from the perspective of others around us, i.e. a holistic approach that is driven to 
work on elimination of such problem, alleviation of the impact of the outbreak. This paper calls all researchers to be more significant efforts to cover the enormous gap in the literature in relevance to frameworks that came bring a resilient multidisciplinary solution for a complex global problem as the COVID-19. Putting close strategic and future foresighted collaborative networks for solving such problem complexity might take us to a new level of human capacity and minimise the dissipated energy we already lost together.

Further, the COVID-19 may be communicate-off like other viruses. However, still many zoonotic viruses might become a human communicable disease just, for example, HIV, the death toll is more than millions in across the world every year. WHO and other leading international entities should encourage a culture of problem-solving than medication research labs, or only optimal isolation or mitigation procedures. Fighting future novel viruses will not be dependent on how much medication, science development, and technological monitoring or control measures we have as these would only postpone the next uncontrollable outbreaks only. The world needs problem solvers that tackle the issue and see the hidden spots inside this world-threatening dilemma.

\section{References}

Buheji, M. (2018). Re-Inventing Our Lives, A Handbook for Socio-Economic "Problem-Solving", AuthorHouse, UK.

Buheji, M. (2020). Visualising Resilient Communities, Authorhouse Publishing, UK.

Christensen, Hansen \& Moore. (1987). Teaching and the Case Method. Harvard Business School, USA.

Drohan, T. (2020). Case Method Problem-Solving in an Uncertain Information Environment: The Novel Coronavirus Threat, Small Wars Journal https://smallwarsjournal.com/jrnl/art/case-method-problem-solving-uncertain-information-en vironment-novel-coronavirus-threat Accessed on: 1/3/2020

Eddy, M. (2020). Germany Tries to Solve a Coronavirus Puzzle With 'Endless Pieces', New York Times, $\quad$ Feb. 27. https://www.nytimes.com/2020/02/27/world/europe/germany-coronavirus.html (Accessed on: $1 / 3 / 2020)$

Emergency and Health Protection Directorate. (2020). Coronavirus: action plan A guide to what you can expect across the UK, 3 March. https://assets.publishing.service.gov.uk/government/uploads/system/uploads/attachment_data/ file/869827/Coronavirus_action_plan_-_a_guide_to_what_you_can_expect_across_the_UK. pdf

Gates, B. (2020). Responding to Covid-19 - A Once-in-a-Century Pandemic? https://doi.org/10.1056/NEJMp2003762

Hemel, D., \& Ouellette, L. (2020). Want a Coronavirus Vaccine, Fast? Here's a Solution. 
March 4, Time. https://time.com/5795013/coronavirus-vaccine-prize-challenge/ (Accessed on: $12 / 3 / 2020)$

Khan, N. (2020). New Virus Discovered by Chinese Scientists Investigating Pneumonia Outbreak. Latest tally of people sickened in Wuhan is 59, with seven in critical condition. https://www.wsj.com/articles/new-virus-discovered-by-chinese-scientists-investigating-pneu monia-outbreak-11578485668

McGrath, R., \& Sargut, G. (2011). Learning to Live with Complexity, September Issue, Harvard Business Review.

Roser, M., \& Ritchie, H. (2020). Coronavirus Disease (COVID-19), Published online at OurWorldInData.org. https://ourworldindata.org/coronavirus (Accessed on: 1/3/2020)

Wetti, P. (2020). The Psychology of Coronavirus vs. Climate Change: Why We Mobilise for One, Not the Other, March 2, Science \& Nature, wttw news. https://news.wttw.com/2020/03/02/psychology-coronavirus-vs-climate-change-why-we-mobi lize-one-not-other (Accessed on: 1/3/2020)

WHO. (2020). Coronavirus disease 2019 (COVID-19): Situation Report - 38. 27 February. World Health Organization. www.who.int/docs/default-source/coronaviruse/situation-reports/20200227-sitrep-38-covid-1 9.pdf?sfvrsn=9f98940c_2 (Accessed on: 12/3/2020)

WHO. (2020b). 2019-nCoV outbreak is an emergency of international concern, 31-01-2020. http://www.euro.who.int/en/health-topics/health-emergencies/international-health-regulations /news/news/2020/2/2019-ncov-outbreak-is-an-emergency-of-international-concern (Accessed on: $1 / 2 / 2020)$

WHO. (2019). Zoonotic disease: emerging public health threats in the Region, http://www.emro.who.int/fr/about-who/rc61/zoonotic-diseases.html(Accessed on: 1/2/2020)

Wu, Y., Chen, C., \& Chan, Y. (2020). The outbreak of COVID-19: An overview. J Chin Med Assoc. 2020 Mar;83(3):217-220. https://doi.org/10.1097/JCMA.0000000000000270

Zhu, N., Zhang, D., \& Wang, W. (2020). A novel coronavirus from patients with pneumonia in China, 2019. New England Journal of Medicine, 382, 727-73. https://doi.org/10.1056/NEJMoa2001017

\section{Copyright}

Copyright for this article is retained by the author(s), with first publication rights granted to the journal. 
This is an open-access article distributed under the terms and conditions of the Creative Commons Attribution license (http://creativecommons.org/licenses/by/4.0/). 